Cahiers d'études italiennes

\title{
Jolles, il Memorabile e il sublime
}

Jolles, Memorable and the Sublime

Jolles, le Mémorable et le sublime

\section{Giuseppe Panella}

\section{(2) OpenEdition}

\section{Journals}

\section{Edizione digitale}

URL: http://journals.openedition.org/cei/3234

DOI: $10.4000 /$ cei.3234

ISSN: 2260-779X

\section{Editore}

UGA Éditions/Université Grenoble Alpes

\section{Edizione cartacea}

Data di pubblicazione: 30 décembre 2016

Paginazione: 195-209

ISBN: 978-2-84310-337-7

ISSN: $1770-9571$

\section{Notizia bibliografica digitale}

Giuseppe Panella, «Jolles, il Memorabile e il sublime», Cahiers d'études italiennes [Online], 23 | 2016, online dal 23 janvier 2017, consultato il 27 mars 2021. URL: http://journals.openedition.org/cei/3234 ; DOI: https://doi.org/10.4000/cei.3234 


\title{
JOLLES, IL MEMORABILE E IL SUBLIME
}

\author{
Giuseppe Panella \\ Scuola Normale Superiore di Pisa
}

\begin{abstract}
Sarei molto contento [...] se, nella sua tecnica di lavoro, lei non partisse dal problema generale, ma da un fenomeno di dettaglio scelto con cura e sicurezza, qualcosa come la storia di una parola o l'interpretazione di un passo. Il fenomeno di dettaglio non deve essere né troppo piccolo né troppo concreto, e non deve mai essere un concetto introdotto da noi o da altri studiosi, ma qualcosa di offerto dall'oggetto stesso.
\end{abstract}

(Erich Auerbach)

\section{Il Memorabile}

La scienza letteraria ha un triplice orientamento. Utilizzando una terminologia fuori uso, possiamo riformulare il concetto dicendo che questa disciplina assolve un compito estetico, storico e morfologico. Volendo esprimerci con maggiore chiarezza, possiamo dire: la scienza letteraria cerca di interpretare un fenomeno letterario in base alla sua bellezza, al suo senso e alla sua forma. Sebbene i tre elementi siano ormai destinati a costituire un'unità triadica, anche in questo caso sembra valido il detto «marciare divisi e colpire uniti». In altri termini, per quanto questi tre fattori si prefiggano di studiare il fenomeno letterario nella sua totalità, ciascuno opera secondo un proprio metodo. Dalla storia delle teorie letterarie si ricava inoltre l'impressione che ogni metodo abbia prevalso in momenti diversi sugli altri due ${ }^{\mathrm{T}}$.

L'incipit dell'opera più celebrata di André Jolles non poteva essere più incisivo, drastico, radicale: la dimensione storica e il possibile impianto storicistico della ricerca ne escono radicalmente ricondotti alla loro dimensione

I. A. Jolles, I travestimenti della letteratura. Saggi critici e teorici (1897-1932), S. Contarini (a cura di), premessa di E. Raimondi, Milano, Bruno Mondadori, 2003, p. 257. 
più propriamente erudita e documentaria (il che non esclude l'uso che lo studioso olandese ne farà anche in seguito e la sua competenza specifica come esperto di dizionari e di raccolte di documenti più propriamente storici). In realtà, anche se Jolles tenderà a privilegiare il metodo morfologico per tentare di costruire una genealogia delle forme letterarie, l'impressione che si ricava dalla lettura di Forme semplici è che la sua analisi della genesi e della costruzione delle forme letterarie debba molto alla scienza del bello come sorge a partire dal primo Romanticismo tedesco e dall'evoluzione del magistero estetico kantiano (l'interesse per Brentano, von Arnim e soprattutto per Johann Georg Hamann lo testimonierebbe). Riassumendo alla fine delle sue riflessioni il frutto delle sue ricerche, Jolles ribadirà la dimensione aggregativa e non solidificata in forma letteraria delle «forme semplici», da lui individuate in un territorio che si muove a metà tra l'antropologia culturale e la pratica della teoria della letteratura propriamente detta.

Delle tre opzioni (il triplice orientamento) individuate da Jolles nella citazione, a me interessa in modo particolare quella legata alla dimensione estetica della sua ricerca: non tanto per trarne indicazioni o proposte in direzione di una possibile retorica o di una coniugazione stilistica, quanto per verificare la potenziale validità di alcune delle categorie da lui individuate come «forme semplici» nell'ambito di una teoria estetica nella quale il bello e il sublime sono percepiti in contrasto.

Ecco dunque le forme che, come abbiamo detto all'inizio, si trovano in uno «stato di aggregazione diverso» rispetto alla letteratura vera e propria e non vengono colte dalle discipline che descrivono la costruzione di un'opera dalle unità e dalle strutture linguistiche fino alla composizione artistica definitiva: forme che (ci pare importante ricordarlo ancora una volta) sono così ancorate al linguaggio da sembrare persino contrarie alla scrittura, l'eterna coscienza del linguaggio. Abbiamo visto che tutte queste forme si realizzano sia nella vita che nel linguaggio, che vengono percepite sia sul piano dell'esistenza che su quello della coscienza: - come sia possibile derivarle ogni volta da una determinata disposizione mentale; come si possa distinguere tra forma semplice pura e forma semplice attualizzata e come da esse scaturisca, in seguito, una forma relativa; e infine come la forma semplice possa ogni volta trasferire il proprio potere a un oggetto, e come l'oggetto si carichi a sua volta del potere della forma. Forse avremmo potuto descrivere tutto ciò in maniera più sistematica. Forse, sin dall'inizio avremmo potuto rilevare e delineare in modo più schematico l'elemento comune a tutte le forme semplici, il loro legame interiore. Di volta in volta, abbiamo tuttavia preferito consentire loro di svilupparsi, per così dire, da sole, ciascuna all'interno del proprio mondo, accennando all'elemento generale che le unisce solo là dove esso emergeva con spontaneità nel corso della singola ricerca² .

2. Ivi, p. 447 . 
A rivestire maggiore importanza sotto il profilo della teoria estetica e dei suoi esiti è a mio avviso ciò che Jolles definisce «disposizione mentale».

Attraverso il raggruppamento prima, e il dispiegamento poi, delle potenzialità originarie e più rilevanti presenti sotto il suo profilo generale, sarà possibile individuare quale modello di riferimento successivo assumano determinate prospettive e possibilità di ricezione del materiale estetico-letterario che saranno da verificare poi in sede di attualizzazione (nel corso della quale, e solo in seguito, esse verranno codificate come costruzione retorica e/o stilistica vigente). In sostanza, la «disposizione mentale» di Jolles si presenta come forma della ricezione dell'atteggiamento estetico predominante nell'atto della sua produzione: non tanto come forma già espressa e codificata, ma come momento in cui essa si condensa intorno a situazioni che il linguaggio presenta nella loro complessità non ancora sistematizzata. Si tratta di un rapporto diretto con il fatto estetico (il suo effetto, o per dirla in termini settecenteschi il suo "affetto») che poi precipiterà, quasi come in una soluzione satura, nel linguaggio. Come scrive Jolles:

Quando dunque sotto l'influsso dominante di una disposizione mentale la pluralità e la molteplicità dell'esistenza e degli avvenimenti concreti si condensano e prendono forma, quando vengono colte dal linguaggio nelle loro unità elementari non divisibili e in architetture linguistiche designano e al tempo stesso significano tale esistenza e tali avvenimenti, diciamo che nasce una «forma semplice». Non è tuttavia facile dare un nome alle creazioni che finora abbiamo chiamato «unità degli avvenimenti» e che sono state assorbite dalla lingua. [...] Le nostre creazioni scatenano senza dubbio qualcosa nella misura in cui, grazie a loro, gli avvenimenti divengono immaginabili a partire da una disposizione mentale ${ }^{3}$.

Di conseguenza, si potrebbe forse sostenere che l'esperienza estetica non si sviluppa a partire dalle forme codificate del linguaggio (letterario o meno), ma a partire da una disposizione mentale che trova solo successivamente una propria sistemazione a livello retorico-stilistico. In tutto quello che colpisce la soggettività, l'oggettivazione del suo effetto avviene a livello linguistico solo dopo che la sua dimensione originaria ha trovato una collocazione adeguata come forma dell'immaginazione creativa e come sua espressione sensibile. Il caso del sublime, nell'accezione che di esso dà la Philosophical Enquiry into the Origin of Our Ideas of Sublime and Beautiful di Edmund Burke (1757), mi sembra particolarmente indicativo di questa potenzialità (e lo si vedrà di seguito nella mia esposizione al momento di ricostruire la natura dell'espressione estetica del Memorabile).

3. Ivi, pp. $287-288$. 
Tra le «forme semplici» analizzate da Jolles spicca a mio avviso, per la sostanziale novità del suo assunto, la forma che Jolles, riprendendo un termine usato per la prima volta da Senofonte, chiama Memorabile (il riferimento è precisamente ai Memorabilia socratici ${ }^{4}$ ). È la qualità del suo "dispositivo mentale» che mi sembra bisognosa di un'analisi approfondita. Che cosa indica e rappresenta ciò che è «memorabile»? Sostanzialmente ciò che spicca per la natura aggregativa dei particolari impiegati per descrivere l'evento cui esso fa riferimento e, di conseguenza, la capacità di aggregazione dei particolari stessi che lo compongono. Proprio all'inizio della sua analisi del Memorabile, Jolles si rifa a un fatto di cronaca come punto di partenza della sua riflessione. È un caso di suicidio - quello del consigliere di commercio Heinrich S., attuato per difficoltà finanziarie non meglio specificate - attraverso il quale Jolles individua il momento in cui un puro e semplice fatto di cronaca (i cui particolari non sembrano in apparenza troppo significativi) diventa Memorabile. Lo diventa non tanto per quello che significa o che manifesta (un uomo di successo si uccide perché la sua condizione è mutata e non è più tale), ma per i particolari dell'evento (al momento del suicidio, la moglie dell'uomo non è in casa, ma all'Opera - dunque il signor S. è solo e derelitto proprio quando avrebbe invece bisogno di vicinanza e conforto; la vicina che si accorge del fatto luttuoso perché ha sentito una detonazione e chiama la polizia perché ne prenda atto è una celebre attrice dell'epoca del muto, Asta Nielsen, e questo particolare viene messo in rilievo non solo per la celebrità della diva, ma anche perché essa è stata la protagonista di opere cinematografiche in cui il suicidio è particolarmente significativo e ricorrente $^{5}$ ). Dunque il suicidio di Heinrich S. viene raccontato in maniera tale da mettere in evidenza non il fatto come tale, ma ciò che lo rende Memorabile: la sua «memorabilità» nasce da ciò che viene collegato all'evento, ovvero per così dire dalla sua aura esterna, e non dal suo essere accaduto in quel modo e in quel luogo. Si tratta di un episodio da cui Jolles ricava una riflessione più generale, come si può vedere da quanto segue:

Proseguiamo il nostro esame. Il vicino avrebbe senz'altro chiamato la polizia e il medico anche se fosse stato uno scapolo qualsiasi oppure la signora X, Y o Z. Ancora una volta, viene invece sottolineato espressamente che la vicina era Asta Nielsen.

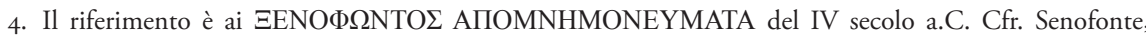
Memorabili, con un saggio di A. Labriola, introduzione, traduzione e note di A. Santoni, Milano, Rizzoli, I989.

5. Per citare soltanto quattro delle moltissime pellicole che hanno visto la Nielsen protagonista, basteranno le sue interpretazioni più specificatamente letterarie: Hamlet, Hedda Gabler (da Ibsen), Signorina Julie (da Strindberg) e Cleopatra, die Herrin des Nils, che culminano tutte in suicidi determinanti per il climax delle opere stesse. 
Quante volte l'eccellente attrice ha assistito o addirittura inscenato un suicidio nella finzione cinematografica, quante volte, sentendo lo sparo di un revolver, si è tappata le orecchie, ha sgranato gli occhi, si è messa le mani nei capelli, si è fatta inquadrare in "primissimo piano». Ed ecco che ora la realtà si affaccia con prepotenza dall'altra parte del muro! Ovviamente, in questo caso non ci domandiamo se la signora Nielsen sia stata contenta di vedere una volta di più il suo nome sul giornale, ma qual è il significato del nostro rapporto. Ancora una volta vediamo come in un evento si trovino espressi due fatti — lo sparo del revolver e la diva cinematografica — che non appaiono legati da alcun nesso causale o esplicativo, ma vengono messi in rapporto tra loro per porre l'accento, attraverso il loro contrasto, sul fatto d'ordine superiore e plasmarlo in modo tale che si imprima nella nostra mente come autonomo ${ }^{6}$.

In sostanza, il Memorabile spicca in virtù della relazione tra gli eventi che lo costituiscono. Esso non sarebbe tale, infatti, se la sua caratteristica fosse quella di essere un puro e semplice episodio storico (in ciò simile a tanti altri avvenuti nel tempo e nello spazio). La sua peculiarità è invece quella di avere qualcosa in più da mostrare e da rendere degno di essere ricordato. Se non ci fossero stati gli sguardi e le movenze sullo schermo della bellissima Asta Nielsen, se non ci fosse stata la circostanza (forse non del tutto secondaria, sotto il profilo dei fatti) dell'assenza della moglie che era andata a teatro, il suicidio sarebbe rimasto quello che era in principio: un trafiletto di cronaca indegno di particolari attenzioni critiche. Riepiloga Jolles:

[...] all'interno dell'evento generale, il ritaglio di cronaca mira a rilevare qualcosa di unico, un insieme che racchiude il senso dell'evento: in questo insieme i dettagli sono disposti in maniera tale che - considerati singolarmente, nei loro rapporti interni e nella loro totalità - sottolineano il senso dell'evento attraverso l'interpretazione, la discussione, il paragone e il confronto ${ }^{7}$.

Il paragone e il confronto creano l'aspettativa rispetto a ciò che diventa Memorabile (la differenza rispetto ad altri casi analoghi, la messa in rilievo della sua qualità unica e non emulabile); l'interpretazione rappresenta il momento della valutazione della qualità dell'evento mentre la discussione apre lo spazio alla messa in discorso delle sue peculiarità e degli elementi che le rendono interessanti o curiose per il lettore o lo spettatore. Il Memorabile spicca perché colpisce, perché crea un effetto scenico, produce un "affetto» in chi legge o assiste allo spettacolo (non è il caso del testo esibito da Jolles, ma la nozione di Memorabile, a mio avviso, potrebbe essere applicata anche alla messinscena teatrale o cinematografica di episodi simili sul piano dell'espressività visiva).

6. A. Jolles, I travestimenti della letteratura, cit., pp. 40I-402.

7. Ibid. 
La messa in scena come espressione significativa di un evento che altrimenti risulterebbe assai meno efficace sotto il profilo della sua proposizione in chiave di attualità o di ricostruzione storica emerge anche dalla narrazione dell'assassinio di Guglielmo d'Orange, che Jolles rievoca durante la sua trattazione della "forma semplice» del Memorabile, riprendendo le osservazioni già espresse nello scambio di lettere con Johan Huizinga che va sotto il nome di Clio e Melpomene ${ }^{8}$.

Il caso della morte di Guglielmo d'Orange è ovviamente molto diverso dal caso del suicidio del consigliere di commercio di cui si era discusso prima. Il principe d'Orange è il simbolo della libertà dei Paesi Bassi e il primo ad averne promosso (e permesso) l'approdo alla libertà repubblicana con la propria attività militare e politica. Nel 1580 sul suo capo fu posta una taglia di venticinquemila corone d'oro, un titolo nobiliare e la totale impunità. Un cattolico francese, Balthasar Gérard, si era convinto della necessità di mettere fine al tradimento perpetrato da Guglielmo e in vista del suo assassinio aveva militato nelle armate del governatore del Lussemburgo (Peter-Ernst I von Mansfeld-Vorderort) per riuscire ad avvicinare il principe olandese. Grazie all'intervento del predicatore calvinista Villiers, il ro luglio 1584 Gérard incontrò Guglielmo per parlargli della propria missione in Francia (doveva consegnare ai francesi il sigillo del governatore Mansfeld in segno di pace) e, una volta arrivato a contatto diretto, gli sparò uccidendolo (il principe olandese gode del poco entusiasmante primato di essere il primo politico europeo a essere stato colpito a morte con una pistola). Gérard morì poi in modo atrocissimo, come accadeva sovente nei casi di uccisione di principi o re, ma non è questo per Jolles a essere «memorabile».

$\grave{\mathrm{E}}$ «memorabile», invece, il modo in cui l'episodio viene raccontato.

Anzitutto è esemplare la perfidia del traditore, che con il danaro datogli dal Principe di Orange per acquistare abiti più decorosi da indossare al posto dei suoi, frusti e logori, compera pistola e pallottole per uccidere il suo benefattore. Poi il timore della giovane moglie di Guglielmo il Taciturno alla vista dell'uomo che lo ucciderà crea una sensazione di inquietudine. Infine l'ambientazione del delitto: le scale che conducono al piano superiore (rispetto al pianoterra dove è situata la sala da pranzo) si interrompono all'altezza di un piccolo vestibolo, dalla cui porticina prospiciente

8. Ivi, pp. 237-252. Nel carteggio con Huizinga (cfr. A. Jolles, J. Huizinga, Clio e Melpomene, trad. it. in A. Jolles, I travestimenti della letteratura, cit., pp. 237-252) si discute anche e a lungo della figura di Giovanna d'Arco e del suo significato letterario, con osservazioni poi riprese in Forme semplici nel lemma dedicato al Mito). 
balza fuori l'assassino e spara. È la descrizione di un agguato. Gli eventi che lo precedono vengono rievocati come se fossero indispensabili, come se fossero vere e proprie cause di quanto accadrà. Scrive Jolles:

Eppure, questi fatti vengono rievocati e svolgono una funzione nel contesto perché spiegano e commentano a loro volta l'episodio, mettendo in rilievo il fatto d'ordine superiore attraverso il confronto e il paragone con gli altri elementi ${ }^{9}$. Vediamo il principe in quel momento preciso e poniamo in relazione l'abbigliamento austero, l'incedere lento e pensoso, la conversazione sugli affari di Stato [tenuta con il borgomastro di Leeuwarden Rombertus van Uylenburg] con la morte repentina del grande uomo, del personaggio indispensabile, ossia con tutto ciò che qui accade. Tali dettagli storici sono in sé privi di importanza. Spingendoci un poco oltre, possiamo addirittura dire che sono tanto irrilevanti da poter essere mutati in qualcos'altro, magari addirittura nel loro esatto contrario. Proviamo a immaginare: il principe, vestito di un farsetto variopinto, lascia con passo leggero la sala da pranzo dove ha scherzato con la giovane moglie Louise de Coligny, quando all'improvviso viene colpito a tradimento. L'immagine è completamente diversa, ma riusciamo a raffigurarcela con la stessa evidenza. La figura del principe è sempre dinanzi a noi, ma ora avvertiamo il contrasto tra il movimento della vita e la morte improvvisa, tra l'allegria del contesto e l'assassinio proditorio ${ }^{\text {To }}$.

In quell' «avvertire il contrasto» tra «il movimento della vita e la morte improvvisa» si annida la possibile emergenza del sublime. Il contrasto tra la vita e la morte viene a configurarsi come un istante assoluto, un momento perfetto di emergenza, una sorta di forma privilegiata di conoscenza della realtà. Anche se gli eventi che costituiscono il Memorabile non sono tali, lo diventeranno nella ricostruzione di chi si porrà di fronte a quell'accadimento come un momento particolare in cui elementi apparentemente poco significativi conteranno poi per sempre.

Jolles analizza il Memorabile come un fatto letterario, non necessariamente e unicamente storico. Ma dalla congerie dei fatti elencati e stratificati emerge una sorta di verità non tanto diversa o più «autentica» della tradizionale lettura storica, quanto una sua dimensione più cogente, capace di colpire l'immaginazione creativa del lettore. Nel Memorabile la letteratura arriva sempre dopo il gesto: la rappresentazione dell'atto (la morte di Guglielmo il Taciturno, la morte del consigliere di commercio S.) si imprime nella mente senza bisogno di artifici retorici (che solo dopo lo consegneranno alla posterità). Afferma ancora Jolles:

9. È questo un elemento centrale nella definizione del Memorabile, e un punto importante di collegamento con il sublime.

Io. A. Jolles, I travestimenti della letteratura, cit., p. 405 . 
Osserviamo come l'avvenimento, che procede in maniera continua e inarrestabile, si cristallizzi in determinati punti, e come, là dove sembra coagularsi e formare una figura, venga colto dalla lingua e riceva una forma letteraria. Vediamo inoltre - ancora una volta, non da un punto di vista storico-filosofico, bensì linguisticoletterario - come tale forma si strutturi per gradi. Tutti i dettagli dell'evento storico, che appartengono a esso e sono obbligati a seguirne il corso e il progresso, all'improvviso mutano direzione, orientandosi verso un fine d'ordine superiore che si presenta come immobile. Presi singolarmente e nel loro complesso, gli elementi correlati mettono in rilievo il fatto d'ordine superiore spiegando, commentando, confrontando e paragonando. Giacché i particolari correlati danno senso al fatto d'ordine superiore e ne vengono a loro volta riempiti di senso, l'insieme si tramuta in una forma che fissa una volta per tutte il significato dell'avvenimento in corso. Nel caso del suicidio del consigliere di commercio S. abbiamo parlato di «ritaglio di giornale». In realtà, non ci riferivamo a qualcosa che ritagliamo con le forbici dalla pagina di un quotidiano, bensì a un elemento che si ritaglia da solo, che si separa dal fatto temporale per divenire autonomo e prendere forma nel giornale. Allo stesso modo, abbiamo definito l'assassinio del principe d'Orange "un ritaglio di storia». Anche in quel caso abbiamo avuto l'impressione di ritagliare qualcosa da un quadro d'insieme, mentre invece abbiamo semplicemente colto qualcosa che si era ritagliato autonomamente dalla storia, e nel quale l'avvenimento storico si era cristallizzato assumendo una forma ${ }^{\text {II }}$.

In conclusione, il Memorabile forma una figura che non ha tanto consistenza come tale (non era stabilito che fosse tale fin dal principio) ma in quanto emergenza, sporgenza all'interno degli eventi che temporalmente si sviluppano attorno a essa. Il "fine d'ordine superiore» che esso assume è il suo valore implicito, che ha bisogno dell'avvolgimento retorico per diventare successivamente esplicito. Per questo motivo, può essere definito "forma semplice» nel senso di Jolles ma anche "figura» nel senso che Auerbach darà a questo termine.

\section{Il sublime}

Quello che caratterizza il Memorabile, tuttavia, non è la sua forma, ma il suo contenuto tangibile, ciò che Jolles chiama la dimensione effettiva del suo essere oggetto della «disposizione mentale». Il Memorabile — aggiunge lo studioso olandese - è concreto (nel significato autentico del termine che viene dal verbo latino concresco, il con-figurarsi insieme dei particolari per la loro comprensione reciproca). I particolari che lo costituiscono sono reali, fatti accaduti e resi mimeticamente. Nella loro effettività risiede 
la forza della sua evidenza presso il lettore e/o lo spettatore. È il concreto della mimesi a rendere il Memorabile capace di imprimersi nella mente e stagliarsi come qualcosa di evidente, di potente, di degno di memoria. I detti di Socrate raccolti da Senofonte cui la titolatura di questa «forma semplice» si ispira non sono, infatti, significativi come tali per la storia della filosofia e del pensiero umano, ma per la significatività della figura del pensatore ateniese così come viene ricordato da chi concretamente l'ha visto e ascoltato (in questo effetto d'eco, il Memorabile si differenzia dalla Leggenda, perché esso non è imitabile come accade a quella forma semplice ma solo individuabile come reale svolgimento di fatti).

I suoi elementi sono significativi e spiccano di per sé, come risulta da quest'altra citazione:

Per ritornare al nostro ultimo esempio di memorabile, ripensiamo alla toga di gala e al passo misurato del principe. Servivano a mettere in risalto l'assassinio e sottolineavano l'omicidio, rendendolo accessibile a un'osservazione distinta, vale a dire concreta. Solamente riempiti come sono da una realtà d'ordine superiore, questi fatti isolati, di per sé irrilevanti, acquistano a loro volta valore. Il principe era vestito e si muoveva ogni giorno in un determinato modo, ma nel flusso dell'avvenimento che abbiamo riconosciuto in lui, tali fatti non balzavano all'occhio, non potevano essere osservati. Solo qui, dove l'avvenimento si cristallizza nella forma, dove tutti i fatti singoli colmano una realtà di ordine superiore e ne vengono colmati, non vediamo più il principe come il rappresentante dell'avvenimento, della ribellione dei Paesi Bassi, bensì in quanto uomo: la tenuta, gli abiti, l'andatura, le azioni delineano il personaggio, a tutto tondo, lo rendono concreto $^{12}$.

I particolari da cui è caratterizzato l'evento che lo vede protagonista, dunque, rendono vera la sua vicenda (e non mitica o leggendaria). Ma c’è qualcosa di più nel Memorabile e nella «disposizione mentale» che lo caratterizza: l'avvenimento narrato acquista significato grazie ai dettagli che ricompongono l'insieme pur rimanendo tali, pur conservando la loro specificità.

Il caso della morte del re visigoto Ataulfo dei Balti, ucciso da Ewerwulf da lui sbeffeggiato per la sua bassa statura (oppure trafitto da Dobbius, uno dei suoi servitori che intende vendicare il suo precedente padrone eliminato dal re visigoto), è, secondo Jolles, un classico esempio di Memorabile (la carriera militare del re procedeva nel migliore dei modi quando egli fu ucciso inopinatamente a causa della vendetta di un suo guerriero troppo spesso da lui sbeffeggiato per la sua altezza) ${ }^{13}$.

I2. Ivi, pp. 408-409.

13. In realtà Jolles racconta la vicenda della morte del re visigoto Ataulfo così come la narrano i fratelli Grimm nelle Deutsche Sagen - il responsabile della morte di Ataulfo fu Sigerico che ne prese il posto per pochissimi giorni (una settimana) per essere a sua volta sostituito da Wallia, più gradito all'imperatore Onorio (fratello di Galla Placidia, forzata moglie di Ataulfo). 
Nella sua narrazione il re muore in maniera improvvisa, e la resa descrittiva del suo omicidio ne concretizza il racconto, altrimenti prevedibile e scontato, tramite un dettaglio specifico: Ataulfo ha il ventre trafitto dal basso in alto dal fendente del suo guerriero che è più basso di lui.

Chi uccide il re è una sorta di nano - quindi chi viene colpito diviene in questo modo una sorta di gigante. Il dettaglio della statura non è anodino, e rivela un'acutezza storica non indifferente pur nell'apparente casualità del dettato. In tal modo, infatti, la morte di Ataulfo diventa Memorabile (come altre morti storicamente celebri e tramandate):

Si era detto precedentemente che, se riuscissimo a individuare nei dettagli la presenza diffusa della disposizione mentale dell'imitatio otterremmo la visione di una parte rilevante del mondo medioevale. Allo stesso modo potremmo dire che ci troveremmo di fronte a una parte considerevole del mondo moderno se riuscissimo a individuare nel dettaglio i punti in cui la disposizione mentale dell'effettivo si manifesta nella vita dell'uomo attuale. In ogni caso, nessuna forma ricorre nell'epoca moderna quanto il memorabile: là dove il mondo venne concepito come un insieme o un sistema di realtà effettive, il memorabile è lo strumento con il quale separare, distinguere e rendere concreto ciò che si presenta come una mescolanza indifferenziata.

Ma questa forma appare tanto familiare e ricorrente all'interno del nostro mondo, che l'epoca contemporanea è forse la meno incline a riconoscerla come tale ${ }^{\mathrm{I4}}$.

Nel dettaglio: è questa la chiave di volta della costruzione jollesiana. Ciò che è effettivo appare composto di momenti precisi e ben definiti che lo rendono proprio per questo attendibile. Ma nella sua puntualità rigorosa il Memorabile è tale solo quando ciò che manifesta e testimonia si mostra come qualcosa che può essere ricondotto alla dimensione di un fatto storicamente conformato o di un assunto che voglia avere queste caratteristiche. Il Memorabile giganteggia perché sembra più reale del reale, più vero del vero, bigger than life. Inoltre non ha bisogno di una sanzione letteraria o retorica per acquisire il suo statuto formale. È già di per sé «attendibile e attestato». Ciò che produce scaturisce da sé e non dalla dimensione letteraria che gli seguirà. La morte di Guglielmo il Taciturno o quella del re Ataulfo sono eventi che spiccano per la loro concretezza e, nello stesso tempo, per la forza espressiva assunta nel loro racconto. La loro verità risulta legata alla loro unicità e al loro carattere, definito nei dettagli. Inoltre nel Memorabile — ammette Jolles — la "forma semplice» tende a sconfinare in un'espressione letteraria che ne utilizza i mezzi:

Anche se in tal modo ci allontaniamo dall'ambito della forma semplice, dobbiamo forse ricordare ancora che le forme artistiche ricorrono sovente ai mezzi del memorabile

I4. A. Jolles, I travestimenti della letteratura, cit., p. 4II. 
quando, per un qualsiasi motivo, mirano a rappresentare un'invenzione come una realtà effettiva, ossia come concreta e attendibile. Nel caso dell'abbigliamento del principe d'Orange, abbiamo già visto come tutti i dettagli concreti si leghino l'uno all'altro e al fatto d'ordine superiore con una relazione significativa. Abbiamo visto (e lo vedremo ogni volta in cui l'avvenimento assumerà la forma del memorabile) come l'allegria che precede un incidente abbia come effetto il contrasto felicitàmalinconia, come la gravità che precede l'incidente stesso generi il presentimento della sventura, come l'incidente produca a sua volta un contrasto se si verifica in una bella mattinata estiva, o una certa armonia se ha luogo durante una tempestosa notte invernale e come, per ripeterlo nuovamente, l'incidente divenga ogni volta concreto nei dettagli e attraverso di essi, come risulti credibile e si imprima nella memoria. Anche quando l'avvenimento non si cristallizza nel memorabile, quando non si ha a che fare con un incidente reale che deve concretizzarsi bensì con un incidente scaturito dall'immaginazione, vediamo tuttavia che, per apparire attendibile, tale incidente prende egualmente la forma del memorabile, circondandosi di singoli elementi correlati tra loro e disposti nell'insieme secondo una relazione significativa ${ }^{15}$.

Di conseguenza, il Memorabile, anche se è la «forma semplice» che si avvicina maggiormente alla forma estetica, non può, quindi, essere assorbito o plasmato dalla pratica letteraria senza una dimensione privilegiata che lo contraddistingua rispetto alle modalità consuete della scrittura e della rappresentazione artistica. Non può dunque essere semplicemente legato alla dimensione del bello (come accade per il fatto artistico conseguente all'evoluzione attualizzata della «forma semplice») ma acquista il carattere del sublime nella sua ricezione soggettiva derivata da Burke ${ }^{\text {I6 }}$. In essa, infatti, il dato oggettivo legato alla forte rilevanza della descrizione del fatto narrato non segue, ma precede, la sua dimensione artistica.

Jolles non considera il sublime una "forma semplice», ma si limita a leggerlo nella sua primaria dimensione retorica, anche se il fatto che ne parli come contraltare rispetto alla satira e alla caricatura potrebbe far pensare che conosca l'Estetica del brutto di Karl Rosenkranz ${ }^{17}$. Tuttavia l'accenno che Jolles ne fa nella trattazione dell'ultima parte delle Einfache Formen, la sezione dedicata allo Scherzo come «forma semplice» della novella, mi pare significativo. Argomenta il critico:

I5. Ivi, p. 4I2

I6. Sulle differenze tra il sublime retorico del cosiddetto Pseudo-Longino (quello del Parisinus Graecus del $\mathrm{X}$ secolo e di altri codici a esso correlati), poi ripreso e divulgato da Boileau, e il sublime nell'accezione psicofisiologica di Burke valgono ancora le considerazioni di S. H. Monk, Il sublime, G. Sertoli (a cura di), trad. it. di R. Garattini, Genova, Marietti, I99I [edizione originale The Sublime, New York, Modern Language Association of America, 1935]. Per il contesto generale in cui vengono assunte queste categorie rimando a G. Panella, Storia del Sublime. Dallo Pseudo-Longino alle poetiche della Modernità, Firenze, Clinamen, 2012.

17. Cfr. K. Rosenkranz, Estetica del brutto [prima edizione italiana 1984; edizione originale Aesthetik des Hässlichen, Königsberg, Bornträger, I853], trad. it. e cura di S. Barbera, introduzione di R. Bodei, Palermo, Aesthetica Edizioni, I994. 
Siamo soliti definire la disposizione mentale da cui scaturisce lo scherzo con il termine greco comico o comicità. Adottando anche qui questa terminologia vorrei fare una precisazione. Proprio come accadde con il concetto di tragico, fu un'estetica filosofica derivata in primo luogo dalle forme artistiche a occuparsi del concetto di comico, giungendo a determinati risultati e classificazioni e definendo addirittura il comico un «valore estraneo all'estetica». Per contro il nostro compito è e continua a essere di tipo morfologico. Ci siamo sempre tenuti lontano dall'estetica e cercheremo di farlo anche qui. In questa sede, non ci preoccupa ciò che, nel senso dell'estetica o nella forma artistica, costituisce il rapporto del comico con quanto è esteticamente tragico, con il sublime, il caratteristico o il bello. Per comico intendiamo una disposizione mentale da cui nasce una forma semplice, nello stesso modo in cui abbiamo inteso il tragico in rapporto alla fiaba ${ }^{18}$.

Nell'ottica delle lezioni di teoria della letteratura da cui deriva il libro del '3o, anche il sublime può essere considerato come un dispositivo mentale piuttosto che come una forma di espressione retorica. Non però nel senso dell'anonimo trattatello Del sublime di età ellenistica, poi riproposto da Boileau nel Seicento, ma in quello, totalmente alternativo alla tradizione classica, del saggio di Edmund Burke, che inaugura la modernità. Se lo snodo dell'argomentazione dell'Anonimo è linguistico-letterario (privilegiando la poesia e le sue forme «naturali» di rappresentazione del «grande animo» dell'autore rispetto alla situazione che raffigura e considera) e si basa essenzialmente su una disciplina morale che si serve della forma come espressione retorica di sentimenti e di situazioni, in Burke la situazione appare rovesciata. La lingua (e, di conseguenza, la sua utilizzazione come parola poetica) non precede ma segue la situazione in cui nasce il sublime. Si è «affetti», colpiti (terrorizzati e affascinati insieme) dall'oggetto o dalla situazione sublime, e solo dopo l'attualizzazione dell'esperienza come evento (narrativo o simbolico) subentra la parola a descriverla compiutamente. La vista del mare in tempesta (classica situazione kantiana), o delle montagne innevate che si ergono apparentemente senza fine nel cielo nuvoloso delle Ande, o delle caverne profonde e buie dove le voci rimbalzano in una eco infinita di suoni minacciosi e suggestivi, sono tutti momenti in cui la lingua non ha alcuna funzione necessitante per produrre l'effetto di sublimità ricercata.

Sono, invece, dispositivi mentali della soggettività investita dalla situazione sublime.

Solo dopo la sua attualizzazione come momento espressivo tramite il linguaggio usato per descriverla la forma del sublime si impone nella sua dimensione letteraria (artistica, pittorica, plastica) e diventa rapporto

I8. A. Jolles, I travestimenti della letteratura, cit., p. 439. 
estetico di confronto tra descrizioni e simbolizzazioni diverse. Il sublime, in realtà, non emerge mai nella sua originarietà concettuale assoluta, ma è sempre una forma del rapporto di differenza tra il bello e le sue possibilità ulteriori. A differenza del tragico, che prevede sempre una sua figuralità definita e stabilita a livello formale, il sublime emerge nel dettaglio, nel momento concreto, nell'effettività del suo essere tale, proprio come avviene nel caso memorabile analizzato nelle pagine di Jolles.

Una controprova dell'utilizzabilità della forma del sublime come espressione figurale che scivola poi in una dimensione stilistica grazie alla sua utilizzazione per differenza è rintracciabile in un'opera che potrebbe sembrare collocarsi al polo opposto di quella di Jolles, vale a dire Mimesis di Erich Auerbach ${ }^{19}$, apparente monumento all'erigenda sociologia della letteratura e poco affine a una visione letteraria di tipo morfologico. Eppure anche nel grande filologo tedesco l'istanza del sublime appare collegata alla dimensione pre-linguistica che anticipa quella stilistica e che scava nella sua successiva proliferazione letteraria. Mettendo in relazione (per differenza, quindi) il Vecchio Testamento con i poemi omerici, Auerbach considera l'esistenza di un livello del sublime presente nella quotidianità descritta in entrambe queste grandi testimonianze del passato, e ritrova in essa la cifra della sua figuralità (in ciò è evidente l'influsso delle hegeliane Lezioni sull'estetica pubblicate da Heinrich Gustav Hotho nel i835 e la temporalizzazione in relazione all'evoluzione degli stili che esse contengono $\left.^{20}\right)$. Su questo punto, Auerbach individua una differenza rilevante nelle forme del sublime esistenti nel periodo storico che analizza:

Dalla più profonda storicità e dalla più profonda mobilità sociale dei testi del Vecchio Testamento dipende infine anche un'ultima distinzione importante: che cioè da essi risulta un concetto dello stile sublime e della sublimità diverso da quello d'Omero. Questi non ha alcun ritegno a immettere il realismo quotidiano nella sublimità tragica, essendo un tale ritegno estraneo al suo stile e con questo inconciliabile: nel nostro episodio della cicatrice [la presenza della cicatrice che rivela Ulisse alla sua vecchia nutrice Euriclea] si vede come la pacifica scena familiare della lavatura dei piedi s'intrecci con il grande, importante e sublime fatto del ritorno. Egli è ancora molto lontano da quella legge della separazione degli stili, più tardi applicata quasi universalmente, per la quale la pittura realistica del quotidiano è inconciliabile

19. Cfr. E. Auerbach, Mimesis. Il realismo nella letteratura occidentale [prima edizione italiana I956; edizione originale Dargestellte Wirklichkeit in der abendländischen Literatur, Bern, München, Francke, I946], trad. it. di A. Romagnoli e H. Hinterhäuser, con un saggio introduttivo di A. Roncaglia, Torino, Einaudi, I999.

20. Su Hegel, il sublime religioso e lo stile classico mi permetto di rimandare al mio Santità del Sublime. Scrivere di Dio in accezione estetico-religiosa, in F. Bazzani et al. (a cura di), Scrivere di Dio, Firenze, Clinamen, 2014, pp. 69-82. 
col sublime e trova il suo posto soltanto nel comico, e, tutt'al più, accuratamente stilizzata, nell'idilliaco ${ }^{21}$.

Che cosa se ne può dedurre in considerazione a quanto è stato detto precedentemente?

Che la sublimità diventa stile, ma non lo è direttamente in quanto è mescolata (nei poemi omerici, secondo quanto afferma Auerbach, ma si potrebbero citare molti altri esempi anche nella classicità - e per farlo, infatti, egli susciterà le ombre di Tacito e di Ammiano Marcellino) alla rappresentazione della vita quotidiana e non costituisce di per sé, ovvero fin da subito, un fatto letterario, ma un'attualizzazione linguistica di cui tenere conto a livello stilistico. Ulisse viene colto nella sua quotidianità (la nutrice gli lava i piedi come si fa con tutti gli ospiti, anche i più umili, ma il fatto diventa un evento per la scoperta della cicatrice che lo identifica) così come i Patriarchi vengono descritti in momenti comuni della loro vita (Abramo, Giacobbe, Giuseppe, Mosè dialogano con Dio in momenti della loro vita quotidiana che diventano solenni soltanto dopo che lo scambio verbale con il divino è avvenuto). Inoltre la dimensione comune della vita risalta maggiormente se attraversata, e trasversalmente tagliata, dal fascio di luce radente del sublime. L'episodio dell'arresto di Pietro detto Valvomeres narrato da Ammiano Marcellino nel cap. 7 del libro XV dei suoi Rerum gestarum libri XXXI definisce in maniera ancora più precisa queste potenzialità del sublime come fatto linguistico, ritrovandole all'interno di una dimensione barocca e straniata della storia come pura e semplice narrazione e non come spiegazione definitiva delle cause e dei suoi eventi. Scrive Auerbach di questa scelta di Ammiano, che ritrova parzialmente anche in Tacito e nel Seneca filosofo morale:

In Tacito, la gravità, la cupezza dello stile storico, nutrita dalla tenebrosità degli avvenimenti narrati, è già talmente pregna di concretezza sensibile, di suggestione dell'orrido, che prende il sopravvento, subito però riafferrata dalla nobile e aspra concisione dello stile, che non permette l'eccesso di tali sopravventi: un esempio fra i tanti, l'esecuzione dei figlioletti di Seiano (Annales, V, 9). In Ammiano soverchia il sensibile pittorico, e questo si è fatto strada entro lo stile illustre non in quanto lo volgarizzi col parlar popolare o comico, ma in quanto l'esagera fuor di misura; la lingua incomincia a dipingere con parole sfolgoranti e con pomposi contorcimenti sintattici una realtà sfigurata, sanguinaria e spettrale [...]. Tutti questi esempi, e molti altri simili, dimostrano che la cosiddetta ampollosità proviene non soltanto dalla tendenza al fuor del comune, ma serve anche, e si direbbe anzitutto, all'evidenza sensibile. Si è costretti a raffigurarsi i fatti. A ciò s'aggiungono le molte comparazioni d'uomini con animali (piacciono particolarmente il serpente e il toro) o di fatti della

2I. E. Auerbach, Mimesis, cit., vol. I, pp. 26-27. 
vita con quelli del teatro o del mondo dei morti. La parola è sempre ricercata, ma l'uso classico adoperava il vocabolo scelto e ricercato per riprodurne nobilmente e in modo generico il sensibile, consentendo la pittura di questo soltanto al poeta, il quale però doveva tenersi lontano dalla realtà presente, per evitare lo stile umile della satira e della commedia; adesso invece, contro l'uso classico, la ricercatezza nello stile sublime della storia serve alla pittura degli avvenimenti presenti. Questa pittura non è però veramente imitativa, rimane sempre lo storico che giudica moralisticamente e parla in stile sublime ed evita le bassure del realismo imitatore, pur impiegando i colori più stridenti ${ }^{22}$.

Si vede bene dunque come il sublime sia una forma di adattamento del fatto narrato alla sua lingua specifica, alla sua espressività tonale. Il dettaglio che ne emerge permette al discorso di far emergere l'originarietà dell'evento e la sua «memorabilità». Non si tratta di un artificio retorico ma di un'insorgenza originaria del linguaggio. Il sublime rende memorabile proprio quell episodio che narra e lo spinge oltre la pura e semplice resa stilistica del suo registro.

Nello stesso modo, a mio avviso, è possibile leggere il rapporto con il Memorabile che interessa a Jolles. Nel realizzare il suo obiettivo, lo studioso olandese mette il suo regime di forme al servizio di una visione della letteratura che affonda le radici nel profondo della lingua, di un'originaria vocazione alla fondazione e alla descrizione della realtà, prima di ogni sua possibile trasformazione stilistica. La «forma semplice» rende così la mimesi verbale frutto di una «disposizione mentale» che viene prima del suo adattamento alla dimensione letteraria e, in tal modo, la attraversa esteticamente, salvandone le potenzialità a essa intrinseche e liberandola dalla pura espressione stilistica che potrebbe schiacciarla in maniera astratta o in vuoto formalismo. Su questo punto, le ricerche così diverse di Jolles e Auerbach trovano forse un possibile punto di convergenza concettuale. 